\title{
Application of the Exponential Of a Matrix Method and the Set of Solutions of a System of Ordinary Differential Equations.
}

\author{
P. N. Habu ${ }^{1}$;D. T. Achaku ${ }^{2}$ \\ ${ }^{1}$ Department of Mathematics, Federal University Lafia, Nigeria. \\ ${ }^{2}$ Department of Mathematics, Federal University Lafia, Nigeria
}

\begin{abstract}
We have established in this paper, that the set of solutions of a system of Ordinary Differential Equations obtained using the exponential of a Matrix Method, can form a Fundamental Matrix. We also discussed when the diagonalizable matrix is not idempotent.
\end{abstract}

Keywords: Diagonalizable, Exponential matrix, matrix, fundamental matrix, idempotent, Ordinary Differential Equations, set of solutions, Wronskian.

\section{Introduction}

In solving a second order non-homogeneous Ordinary Differential equation we have many methods, namely:- Method of Undetermined Coefficient also called Method of Judicial Guessing, Method of Variation of Parameters, Inverse D-operator Method, etc [1].

However, as the order of the ordinary differential equations goes higher it becomes more tedious to solve this system of differential equations. In this case we have to reduce each into a system of $\mathrm{n}$ first order linear ordinary differential equations.

Jervin Zen Lobo and Terence Johnson [1] gave the solution of a system of ordinary differential equations, using Exponential of a Matrix Method. They did not discuss special cases of the diagonalizable matrix that is not idempotent. They only considered the case of Nilpotent Matrix. They also did not apply the concept of Wronskian, to show that the set of solutions of the ordinary differential equations can form a fundamental matrix, so as to agree with other Methods, that the set of solutions can form a fundamental Matrix. Jervin Zen Lobo and Terence Johnson [1], did not show that the set of solutions obtained using the exponential of a matrix in solving a system of ordinary differential equations, form a fundamental Matrix, if the Wronskian is not equal to zero. In this paper, we have shown that the set of solutions obtained using the exponential of a matrix in solving a system of ordinary differential equations can form a fundamental matrix.

\section{Proposed Method}

We consider a system of difference equations, in order to later write it as a Matrix, $\mathrm{A}$, where the solution $\mathrm{U}_{\mathrm{k}}=\mathrm{A}^{\mathrm{k}} \mathrm{U}_{\mathrm{o}}$,

depends on the power of. It is equally true for ordinary differential equations, where the solution of $\frac{\mathrm{du}}{\mathrm{dt}}=$ $\mathrm{AU}$ is given by

$$
\mathrm{U}(\mathrm{t})=\mathrm{ce}^{-\mathrm{At}}
$$

depends on the exponential of A [1], where $\mathrm{C}$ is constant. From linear Algebra, we know that for any matrix A, the exponential of $\mathrm{A}$ is

$$
\mathrm{e}^{\mathrm{A}}=1+\mathrm{A}+\frac{\mathrm{A}^{2}}{2 !}+\frac{\mathrm{A}^{3}}{3 !}+\ldots
$$

if $\mathrm{A}$ is idempotent.

We can see that equation (3) imitates the power series definition of

$\mathrm{e}^{\mathrm{x}}=1+\mathrm{x}+\frac{\mathrm{x}^{2}}{2 !}+\frac{\mathrm{x}^{3}}{3 !}+$

Which always converges, therefore equation (3) will also always converges. Since matrix A is now considered idempotent, diagonalizable and of degree $\mathrm{n}, \mathrm{n}=1,2,3, \ldots$, we shall use the exponential of a matrix in the following way [1].

Step 1: Write down the given system of ordinary differential equations as a matrix, A.

Step 2: Find the eigenvalues and eigenvectors of matrix, A. From another matrix $S$ whose columns are eigenvectors of $\mathrm{A}$.

Step 3: Compute $S^{-1} A S$ which will be the diagonal. For example, $S^{-1} A S=\operatorname{diag}(\alpha, \beta, \Upsilon)$ where $\alpha, \beta$ and $\Upsilon$ are constants.

Step 4: Now, write $A=S \operatorname{diag}(\alpha, \beta, \Upsilon) S^{-1}$. Hence, finally, $\mathrm{e}^{\mathrm{tA}}=\mathrm{S}\left(\mathrm{e}^{\mathrm{t} \alpha}, \mathrm{e}^{\mathrm{t} \beta}, \mathrm{e}^{\mathrm{t} \gamma}\right) \mathrm{S}^{-1}$. 
Step 5: using the result that the general solution of $\frac{d u}{d t}=A U$ is $U(t)=e^{t A} c$, therefore the solution of the system of the ordinary differential equations is $\mathrm{e}^{\mathrm{tA}} \mathrm{C}$, obtained from step 4.

\section{The set of solutions of the System of ordinary differential equations.}

Theorem 1: If an $\mathrm{n} \times \mathrm{n}$ matrix $\mathrm{A}$, has a basis of eigenvectors, then $\mathrm{D}=\mathrm{S}^{-1} \mathrm{AS}$

Is diagonal with the eigenvectors of $\mathrm{A}$ as entries on the main diagonal and $\mathrm{S}$ is a matrix with eigenvectors as column vectors. Also, $\mathrm{D}^{\mathrm{m}}=\mathrm{SA}^{\mathrm{m}} \mathrm{S}^{-1}$, for $\mathrm{m}=1,2,3$,

\section{Proof:}

Let $\mathrm{s}_{1}, \mathrm{~s}_{2}, \mathrm{~s}_{3}, \ldots, \mathrm{s}_{\mathrm{n}}$ be a basis of eigenvectors of $\mathrm{A}$ for $\mathrm{R}^{\mathrm{n}}$, and the corresponding eigenvalues of $\mathrm{A}$ be $\lambda_{1}, \lambda_{2}$, $\lambda_{3}, \ldots, \lambda_{\mathrm{n}}, \quad$ respectively. So that $\mathrm{As}_{1}=\lambda_{1} \mathrm{~s}_{1}, \mathrm{As}_{2}=\lambda_{2} \mathrm{~s}_{2}, \ldots, \mathrm{As}_{\mathrm{n}}=$ $\lambda_{\mathrm{n}} \mathrm{s}_{\mathrm{n}}$

then $S=\left[s_{1}, s_{2}, \ldots, s_{n}\right]$ has rank $n$ since the rank $r$, of a matrix A equals the maximum number of linearly independent column vectors, and $\mathrm{A}$ and $\mathrm{A}^{\mathrm{T}}$ have the same rank.

$$
\left.\begin{array}{rl}
\text { So, A S }= & A \\
& {\left[s_{1}, s_{2}, \ldots, s_{n}\right]} \\
& =\left[A s_{1}, A s_{2}, \ldots, A s_{n}\right] \\
& =\left[\lambda_{1} s_{1}, \lambda_{2} s_{2} \lambda s_{2}, \ldots, \lambda_{n} s_{n}\right] \\
& =S D
\end{array}\right\}
$$

The fourth equality in (8) follows direct calculation, the third uses, $\mathrm{SK}=\lambda \mathrm{k}$ sk.

The second equality results if we note that the first column of AS, is A times the first column of $S$, which is $s_{1}$ and so on. For instance, when $n=2$, we have, $s_{1}=\left[s_{11}, s_{21}\right], s_{2}=\left[s_{12}, s_{22}\right]$ so that

$$
\begin{aligned}
& A S=A\left(s_{1} \quad s_{2}\right) \\
& =\left(\begin{array}{ll}
a_{11} & a_{12} \\
a_{21} & a_{12}
\end{array}\right)+\left(\begin{array}{ll}
s_{11} & s_{12} \\
s_{21} & s_{22}
\end{array}\right) \\
& =\left(\begin{array}{ll}
a_{11} s_{11}+a_{12} s_{12} & a_{11} s_{12}+a_{12} s_{22} \\
a_{21} s_{11}+a_{22} s_{21} & a_{21} s_{12}+a_{22} s_{22}
\end{array}\right) \\
& =\left(\begin{array}{lll}
A s_{1} & A s_{2}
\end{array}\right)
\end{aligned}
$$

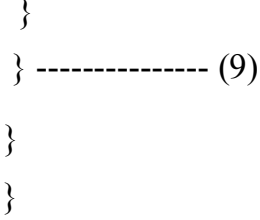

If we multiply (9) by $\mathrm{S}^{-1}$ from the right, we get (8), since it is a similarity transformation. D has the same eigenvalues as $\mathrm{A}$ and (9) follows if we note that:-

$$
\begin{aligned}
& \mathrm{D}^{2} \begin{aligned}
& =\mathrm{DD} \\
& =\left(\mathrm{SAS}^{-1}\right)\left(\mathrm{SAS}^{-1}\right) \\
& =\mathrm{SA}\left(\mathrm{S}^{-1} \mathrm{~S}\right) \mathrm{AS}^{-1} \\
& =\mathrm{SA} \mathrm{AS}^{-1} \\
& =\mathrm{SA} \mathrm{A}^{2} \mathrm{~S}^{-1}
\end{aligned} \\
& \text { Hence, } \mathrm{D}^{\mathrm{m}}=\mathrm{SA}^{\mathrm{m}} \mathrm{S}^{-1}
\end{aligned}
$$

Theorem 2:- The solution of the homogenous system of differential equations $\frac{\mathrm{du}}{\mathrm{dt}}=\mathrm{AU}+\mathrm{g}$ where,

$A=\left(\begin{array}{ccccc}a_{11} & a_{12} & a_{13} & \ldots & a_{1 n} \\ a_{21} & a_{22} & a_{23} \ldots \ldots & a_{2 n} \\ \vdots & \vdots & \vdots & \vdots \\ a_{n 1} & a_{n 2} & a_{n 3} & \cdots & a_{n n}\end{array}\right), U=\left(\begin{array}{c}u_{1} \\ u_{2} \\ \vdots \\ u_{n}\end{array}\right)$ and $g=\left(\begin{array}{c}g_{1} \\ g_{2} \\ \vdots \\ g_{n}\end{array}\right)$

On some interval $\mathrm{I}$ is a linearly independent set of $\mathrm{n}$ solutions, $\mathrm{U}^{(1)}, \mathrm{U}^{(2)}, \mathrm{U}^{(3)}, \ldots ., \mathrm{U}^{(\mathrm{n})}$ if and only if the determinant is not equal to zero.

Proof : Let $U=c_{1} U^{(1)}+c_{2} U^{(2)}+\ldots \ldots+U^{(n)}$

be a linear combination of $\frac{\mathrm{du}}{\mathrm{dt}}=\mathrm{AU}$ on some interval $\mathrm{I}$. it can be shown that if $\mathrm{a}_{\mathrm{jk}}(\mathrm{t})$ in $\frac{\mathrm{du}}{\mathrm{dt}}=\mathrm{AU} \sin$ are continous on I then $\frac{d u}{d t}=A U$ has a basis of solutions on I. We write these $n$ solutions as an $n \times n$ matrix in the form

$U=\left[\mathrm{U}^{(1)}, \mathrm{U}^{(2)}, \ldots \ldots, \mathrm{U}^{(\mathrm{n})}\right]$

The Wronskian, $\mathrm{W}(\mathrm{U})$ is

$\mathrm{W}\left(\mathrm{U}^{(1)}, \mathrm{U}^{(2)}, \ldots \ldots, \mathrm{U}^{(\mathrm{n})}\right)=\left|\begin{array}{cccc}\mathrm{u}_{1}^{(1)} & \mathrm{u}_{1}^{(2)} & \mathrm{u}_{1}^{(3)} \ldots \ldots & \mathrm{u}_{1}^{(\mathrm{n})} \\ \mathrm{u}_{2}^{(1)} & \mathrm{u}_{2}^{(2)} & \mathrm{u}_{2}^{(3)} \ldots \ldots & \mathrm{u}_{2}^{(\mathrm{n})} \\ \vdots & \vdots & \vdots \ldots \ldots \ldots & \vdots \\ \mathrm{u}_{\mathrm{n}}^{(1)} & \mathrm{u}_{\mathrm{n}}^{(2)} & \mathrm{u}_{\mathrm{n}}^{(3)} \ldots \ldots & \mathrm{u}_{\mathrm{n}}^{(\mathrm{n})}\end{array}\right|$

If $\mathrm{W}(\mathrm{U}) \neq 0$, then $\mathrm{U}=\mathrm{c}_{1} \mathrm{U}^{(1)}+\mathrm{c}_{2} \mathrm{U}^{(2)}+\ldots \ldots+\mathrm{c}_{\mathrm{n}} \mathrm{U}^{(\mathrm{n})}$ forms a basis and $\mathrm{U}=\left[\mathrm{U}^{(1)}, \mathrm{U}^{(2)}, \ldots \ldots, \mathrm{U}^{(\mathrm{n})}\right]$ is called Fundamental Matrix. 


\section{Application Of The Concept Of Wronskian [2][4]}

Since the general solution of $\frac{\mathrm{du}}{\mathrm{dt}}=\mathrm{AU}$ as established using the exponential of Matrix, $\mathrm{A}$, in the solution of system of ordinary differential equations, is $\mathrm{U}(\mathrm{t})=\mathrm{Ce}^{\mathrm{At}}$, therefore the $\mathrm{n}$ solutions of $\frac{\mathrm{du}}{\mathrm{dt}}=\mathrm{AU}$ are $\mathrm{U}^{(1)}, \mathrm{U}^{(2)}, \ldots \ldots$, $\mathrm{U}^{(\mathrm{n})}$ on Some interval I. it ollows that $\mathrm{U}(\mathrm{t})=\mathrm{c}_{1} \mathrm{U}^{(1)}+\mathrm{c}_{2} \mathrm{U}^{(2)}+\mathrm{c}_{3} \mathrm{U}^{(3)}+\ldots \ldots+\mathrm{c}_{\mathrm{n}} \mathrm{U}^{(\mathrm{n})}$

We now write these $n$ solutions of $\frac{\mathrm{du}}{\mathrm{dt}}=\mathrm{AU}$ on some interval $\mathrm{I}$ as columns of an $\mathrm{n} \times \mathrm{n}$ matrix $\mathrm{U}=\left[\mathrm{U}^{(1)}, \mathrm{U}^{(2)}, \mathrm{U}^{(3)} \ldots \ldots, \mathrm{U}^{(\mathrm{n})}\right]$

Where $U^{(r)}=\left[U_{1}^{(r)} U_{2}^{(r)} U_{3}^{(r)} \ldots U_{n}^{(r)}\right], r=1,2,3, \ldots, n$.

The Wronskian,

$$
\mathrm{W}\left(\mathrm{U}^{(1)}, \mathrm{U}^{(2)}, \ldots \ldots, \mathrm{U}^{(\mathrm{n})}\right)=\left|\begin{array}{ccccc}
\mathrm{u}_{1}^{(1)} & \mathrm{u}_{1}^{(2)} & \mathrm{u}_{1}^{(3)} & \ldots . & \mathrm{u}_{1}^{(\mathrm{n})} \\
\mathrm{u}_{2}^{(1)} & \mathrm{u}_{2}^{(2)} & \mathrm{u}_{2}^{(3)} & \ldots & \mathrm{u}_{2}^{(\mathrm{n})} \\
\vdots & \vdots & \vdots & \ldots \ldots & \vdots \\
\mathrm{u}_{\mathrm{n}}^{(1)} & \mathrm{u}_{\mathrm{n}}^{(2)} & \mathrm{u}_{\mathrm{n}}^{(3)} & \ldots . & , \mathrm{u}_{\mathrm{n}}^{(\mathrm{n})}
\end{array}\right|
$$

If $\mathrm{W}(\mathrm{U}) \neq 0$, then this set of solution forms Fundamental system of solutions (basis) of the homogenous ordinary differential equation, $\frac{\mathrm{du}}{\mathrm{dt}}=\mathrm{AU}$ and $\mathrm{U}$ in equation (14) is called a Fundamental Matrix.

Example 1: Diagonalizable Matrix

\section{Examples}

Let $A=\left(\begin{array}{ccc}7.3 & 0.2 & -3.7 \\ -11.5 & 1 & 5.5 \\ 17.7 & 1.8 & -9.3\end{array}\right)$

To diagonalise $A$, the characteristic determinant of $A$ gives the characteristics equation $-\lambda^{3}-\lambda^{2}+12 \lambda=0$

The roots of $A$ are therefore $\lambda_{1}=3, \lambda_{2}=-4$, and $\lambda_{3}=0$.

From $(A-\lambda I) U=0$ with $\lambda=\lambda_{1}, \lambda_{2}, \lambda_{3}$, we find the eigenvectors and then $U^{-1}$ The results are

$\mathrm{U}=\left(\begin{array}{ccc}-1 & 1 & 2 \\ 3 & -1 & 1 \\ -1 & 3 & 4\end{array}\right), \quad \mathrm{U}^{-1}=\left(\begin{array}{ccc}-0.7 & 0.2 & 0.3 \\ -1.3 & -0.2 & 0.7 \\ 0.8 & 0.2 & -0.2\end{array}\right)$

$\mathrm{UAU}^{-1}$

$=\left(\begin{array}{ccc}-0.7 & 0.2 & 0.3 \\ -1.3 & -0.2 & 0.7 \\ 0.8 & 0.2 & -0.2\end{array}\right)\left(\begin{array}{ccc}7.3 & 0.2 & -3.7 \\ -11.5 & 1 & 5.5 \\ 17.7 & 1.8 & -9.3\end{array}\right)\left(\begin{array}{ccc}-1 & 1 & 2 \\ 3 & -1 & 1 \\ -1 & 3 & 4\end{array}\right)$
$=\left(\begin{array}{ccc}3 & 0 & 0 \\ 0 & -4 & 0 \\ 0 & 0 & 0\end{array}\right)$

Example 2: Calculating the Wronskian [5],

The system of ordinary differential equations [1],

$\left.\begin{array}{l}\frac{d x}{d t}=x-y+4 z \\ \frac{d y}{d t}=3 x-2 y+z \\ \frac{d z}{d t}=2 x-y+z z\end{array}\right\}$

using the exponential of a matrix Method, has the general solution $X=e^{t A} c$, which can be written as

$\left(\begin{array}{l}x \\ z\end{array}\right)=\frac{1}{6}\left[\begin{array}{ccc}e^{t}+3 e^{3 t}+2^{-2 t} & -2 e^{t}+2 e^{-2 t} & 3 e^{t}+3 e^{2 t}-6 e^{-2 t} \\ 14 e^{-t}+6 e^{3 t}-2 e^{-2 t} & 8 e^{t}-2 e^{-2 t} & 12 e^{t}+6 e^{3 t}+6 e^{-2 t} \\ -e^{t}+3 e^{3 t}-2 e^{-2 t} & 2 e^{t}-2 e^{-2 t} & -3 e^{t}+3 e^{3 t}+6 e^{-2 t}\end{array}\right]\left(\begin{array}{c}c_{1} \\ c_{2} \\ c_{3}\end{array}\right)$

Where $c_{1}, c_{2}, c_{3}$ are arbitrary constants. The Wronskian, $\mathrm{W}(\mathrm{X})$ of equation (21) is

$$
\begin{aligned}
W(x) & =\left|\begin{array}{ccc}
\left(e^{t}+3 e^{3 t}+2^{-2 t}\right) c_{1} & \left(-2 e^{t}+2 e^{-2 t}\right) c_{2} & \left(3 e^{t}+3 e^{2 t}-6 e^{-2 t}\right) c_{3} \\
\left(14 e^{-t}+6 e^{3 t}-2 e^{-2 t}\right) c_{1} & \left(8 e^{t}-2 e^{-2 t}\right) c_{2} & \left(12 e^{t}+6 e^{3 t}+6 e^{-2 t}\right) c_{3} \\
\left(-e^{t}+3 e^{3 t}-2 e^{-2 t}\right) c_{1} & \left(2 e^{t}-2 e^{-2 t}\right) c_{2} & \left(-3 e^{t}+3 e^{3 t}+6 e^{-2 t}\right) c_{3}
\end{array}\right| \\
& =f\left(e^{-t}, e^{-2 t}, e^{t}, e^{3 t}\right)
\end{aligned}
$$

$\mathrm{W}(\mathrm{x}) \neq 0$ for all $\mathrm{t}$ in the interval $\mathrm{I}$, that is, $\mathrm{X}$ the set of solutions of the system of ordinary differential equations is a Fundamental Matrix and the set $\mathrm{X}$ of the solutions is linearly independent.

\section{Discussing Special Cases Of The Matrix, $A$.}

(a) When $\mathrm{A}$ is a nilpotent matrix, then for $\mathrm{e}^{\mathrm{A}}=1+\mathrm{A}+\frac{\mathrm{A}^{2}}{2 !}+\frac{\mathrm{A}^{3}}{3 !}+\ldots$, there exist a number $\mathrm{m}$ such that $\mathrm{A}^{\mathrm{m}}=0$. Therefore the series will terminate at some point. 
(b) When $\mathrm{A}$ is an idempotent matrix, the series $\mathrm{e}^{\mathrm{A}}$ does not terminate for any $\mathrm{A}^{\mathrm{m}}$, because $\mathrm{A}^{\mathrm{m}}=\mathrm{A}$ for an idempotent matrix.

(c) If $\mathrm{A}$ is a symmetric matrix, i.e $\mathrm{A}^{\mathrm{T}}=\mathrm{A}$, it means that $\mathrm{e}^{\mathrm{A}}=\mathrm{e}^{\mathrm{A}^{\mathrm{T}}}$.

(d) If $A$ is a skew- symmetric matrix, i.e $A^{T}=-A$, therefore $e^{-A}=e^{A^{T}}$.

\section{Conclusion}

In using the method of exponential of a matrix, it is clear that we need the matrix to be idempotent and diagonalizable and that the set of solutions obtained can form a Fundamental Matrix.

The concept of a fundamental matrix is used for construction site applications in civil Engineering, by estimating the fundamental matrix, to determine the epipolar geometry between a pair of images or video frames [6]. The estimation of fundamental matrix is also one of the most crucial steps in many computer vision applications such as 3D reconstruction, autocalibration and motion segmentation [7]. It is therefore hoped that this research will stimulate the interest of more investigators on the concept of fundamental matrix.

[1]. Jervin Zen lobo and Terence Johnson,

\section{References}

[2]. Solution of Differential Equations Exponential of a Matrix, IOSR Journal of Mathematics, e-ISSN 2278 - 5728, volume 5, Issue 3, (Jan - Feb 2013) pp $12-17$.

[3]. Erwin Kreyszig,

[4]. Advanced Engineering Mathematics, $10^{\text {th }}$ Edition, pp 75- 78.

[5]. Dennis G. Zill,

[6]. A First Course In Differential Equations with Applications. Pp 124-125,401.

[7]. George B. Arenk and Hans J. Webber,

[8]. Advanced Mathematics for Physicists, $6^{\text {th }}$ Edition. Pp $577-580$.

[9]. Allan Jeffrey,

[10]. Mathematics for Engineers and Scientists, $3^{\text {rd }}$ Edition Publisher, ELBS, UK. Pp $603-65$

[11]. Gauri M. Iog, Habib Fathi,

[12]. Automated Computational of the fundamental matrix for vision based construction site applications. Journal Advanced Engineering Informatics Archive Volume 25 Issue 4, October 2011, Pages 725-735.

[13]. Xuelian Xiao,

[14]. Computer Application and System Modeling (ICCASM), 2010 International Conference [Volume 7] Date of conference: 22-24 Oct. 2010, Pages: V7 400-V7 404. E-ISBN: 978-1-4244-7237-6. Print ISBN: 978-1-4244-7235-2. 